\title{
Cellular response markers and cytokine gene expression in the central nervous system of cattle naturally infected with bovine herpesvirus 5
}

\author{
T.C. Cardoso ${ }^{\text {a,* }}$, H.L. Ferreira ${ }^{\text {b }}$, L.H. Okamura ${ }^{a}$, T.P. Giroto ${ }^{a}$, B.R.S.M. Oliveira ${ }^{\text {a }}$, C.U.F. Fabri ${ }^{\text {a }}$, \\ R. Gameiro ${ }^{\text {a }}$, E.F. Flores ${ }^{\mathrm{c}}$ \\ a Laboratory of Animal Virology and Cell Culture, College of Veterinary Medicine, Univ - Universidade Estadual Paulista, Araçatuba, São Paulo 16050-680, \\ Brazil \\ ${ }^{\mathrm{b}}$ Department of Veterinary Medicine, FZEA-USP-University of Sao Paulo, Av. Duque de Caxias Norte, 225, Pirassununga, São Paulo 13635-900, Brazil \\ c Virology Section, Federal University of Santa Maria, Santa Maria, Rio Grande do Sul 97115-900, Brazil
}

\section{A R T I C L E I N F O}

\section{Article history:}

Accepted 20 November 2016

\section{Keywords:}

Cattle

Inflammatory response

Viral meningoencephalitis

\begin{abstract}
A B S T R A C T
The present study reports an investigation on the phenotype of inflammatory and immune cells, cytokine and viral gene expression in the brains of cattle naturally infected with bovine herpesvirus 5 (BHV5). Brain sections of 38 affected animals were analysed for the nature and extent of perivascular cuffs in the Virchow-Robin space and parenchyma.

Histopathological changes were severe in the olfactory bulbs (Obs), hippocampus, piriform, frontal, temporal and parietal cortices/lobes and were characterized by inflammatory infiltrates in VirchowRobin spaces. The histopathological changes correlated positively with the distribution of BHV5 antigens $(r=0.947 ; P<0.005)$. Cells of CD3+ phenotype were predominant in areas with severe perivascular cuffs. Viral antigens and genomic viral DNA were detected in the Obs and piriform lobe, simultaneously $(r=0.987$; $P<0.005)$. Similarly, pro-inflammatory cytokine genes INFG, IL2, TNF and LTBR were expressed in the same brain areas $(P<0.005)$. These results provide important information on the inflammatory and immunological events accompanying BHV5 neurological infections. Our findings provide the first evidence for increased immune activation followed by inflammatory cytokine expression, positively correlated with viral replication in the cranial areas of the brain. Taken together, these results suggest that the host immune response and inflammation play a crucial role in the pathogenesis of acute encephalitis by BHV5 in cattle.
\end{abstract}

(c) 2016 Elsevier Ltd. All rights reserved.

\section{Introduction}

Bovine herpesvirus 5 (BHV5) belongs to the family Herpesviridae, subfamily Alphaherpesvirinae, genus Varicellovirus (Davison et al., 2009; Davison, 2010; Del Medico Zajac et al., 2010). A hallmark of the biology of alphaherpesviruses is the lifelong latent infection established after acute infection, providing a means for viral reactivation, transmission and spread (Perez et al., 2002; Jones, 2003; Lovato et al., 2003; Jones et al., 2011; St Leger and Hendricks, 2011). The active viral replication during acute infection and after viral reactivation frequently results in neurological disease characterized by tremors, nystagmus, tooth grinding, bruxism, ptyalism, tongue protrusion, circling, ataxia, paddling and, eventually, death (Del Medico Zajac et al., 2010; Marin et al., 2014).

Several reports have described experimental inoculations of BHV5 to reproduce clinical encephalitis and produced variable results (Perez et al., 2002; Del Medico Zajac et al., 2010; Machado et al., 2013). Neurologic signs and microscopic lesions observed during

\footnotetext{
* Corresponding author.

E-mail address: tcardoso@fmva.unesp.br (T.C. Cardoso).
}

acute phase of BHV5 infection after experimental and/or natural field outbreaks were considered similar (Perez et al., 2002). Moreover, during acute infection, BHV5 would access mostly the rostral areas indicating that viral invasion of the brain occurs more directly along olfactory neurons (Favier et al., 2014). In summary, the types of neural damage produced after BHV5 infection of the brain have been described as neuronal degeneration and inflammatory perivascular cuffs with different degrees of severity (Machado et al., 2013). However, due to the variability of these histopathological patterns, the characterization of lymphocyte recruitment after natural BHV5 infection remains unclear.

Cytokine levels are markers of the inflammatory cascade in the CNS, in which the immune system plays an essential role in the homeostasis (Yshii et al., 2015). A number of neurotropic viruses induce the loss/disruption of the blood-brain barrier (BBB), including Nipah virus, rabies virus, West Nile virus, mouse adenovirus type 1 and herpes simplex virus (HSV1; Sarma, 2014). A similar mechanism has been recently proposed for BHV5 in rabbits (Machado et al., 2013). Inflammatory cytokines or chemokines, such as gamma interferon (IFN- $\gamma$ ), interleukin 8 (IL-8), tumour necrosis factor alpha (TNF$\alpha$ ) and IL-1 $\beta$, may facilitate virus entry into the CNS by inducing a breakdown in the BBB (Franco and Fernández-Suárez, 2015). Major 
sources of these cytokines include the microglial CNS cells or infiltrating lymphocytes, which move towards the site of infection via chemokine-mediated cell trafficking upon the breakdown of the BBB (Yshii et al., 2015). However, the inflammatory response is an essential component of the host immune system, as observed in HSV1 infection, where $\mathrm{T}$ and $\mathrm{B}$ lymphocytes remain activated for a long time (Jones, 2003). Lymphocyte T cells also induce the biological activation of astrocytes in experimental and natural cases of BHV5 infection (Cardoso et al., 2010; Machado et al., 2013). Moreover, IFN$\gamma$, produced by a subset of $\mathrm{T}$ cells, has been described as a potent activator of astrocytes (Xie and Yang, 2015).

Since many aspects of BHV5-induced neuroinflammation remain obscure, the present study was designed to investigate the phenotype of inflammatory and immune cells, cytokine and viral gene expression in the brains of cattle naturally infected with bovine herpesvirus 5 (BHV5), correlating them with the distribution of viral antigens and genome.

\section{Materials and methods}

\section{Animals and ethical statement}

Brains from 38 cattle with positive diagnoses for acute BHV5 neurological infection and from 10 healthy cattle were obtained from routine pathological diagnostics at the Laboratory of Animal Virology of University of São Paulo state, Brazil. All applicable institutional guidelines for the care and use of animals were followed (CEEA 2015/09765). In each animal, the whole brain was sampled, sectioned into two halves and divided into 12 regions, as follows: rostral areas: Ob (olfactory bulbs and rostral olfactory peduncles), $\mathrm{Pl}$ (Piriform lobe), $\mathrm{H}$ (hippocampus), Fc (frontal cortex), Tc (temporal cortex), Pc (parietal cortex); caudal areas: Oc (occipital cortex), T (thalamus), M (mesencephalon), P (pons), Mob (medulla oblongata), Sc (cervical spinal cord). For each region from each brain half, two fragments of approximately $12.5 \mathrm{~mm}$ were stored in RNA later-ICE (Ambion, Life Technologies) at $-86^{\circ} \mathrm{C}$ for molecular analysis, and immersed in $10 \%$ formalin for histopathological and immunohistochemical analyses in parallel (Vogel et al., 2003; Marin et al., 2015).

\section{Histochemical and immunohistochemical investigations}

Sections from both brain halves, embedded in paraffin wax, in serial sections, $3-\mu \mathrm{m}$ thick, were submitted to haematoxylin and eosin staining following standard procedures (Ferrari et al., 2007). The numbers of perivascular cuffs were counted by two different pathologists masked to the experimental design, and they were classified as mild (increase in cell nuclei or incomplete ring of cell nuclei within the Virchow-Robin space), moderate (complete ring of cells in the Virchow-Robin space) or severe (two or more complete cell layers in the Virchow-Robin space).

BHV5 antigen levels and the inflammatory cell phenotype were determined according to previously reported methods, with some modifications (Cardoso et al., 2010). The LSAB + Kit was used according to the manufacturer's recommendations (K0690 Dako). BoHV5 monoclonal antibody (BHV5 2F9) was used at a dilution of $1: 50$ in $1 \%$ bovine serum albumin (BSA, Sigma-Aldrich). T lymphocytes were identified and counted using the mouse monoclonal antibody anti-CD3 diluted at 1:100 in 1\% BSA. For B-lymphocytes, mouse anti-CD45R/B220 antibody (Sigma-Aldrich) diluted at $1: 50$ in $1 \%$ BSA was applied. Epitope retrieval was performed by immersion of slides in a solution of $0.05 \%$ trypsin (Sigma-Aldrich). The reaction was performed according to the instructions of the LSAB + Kit (Dakocytomation). Finally, the slides were counterstained with Harris's haematoxylin and mounted. Negative control sections were prepared by replacing the primary antibodies with $1 \%$ BSA (Sigma-Aldrich; Machado et al., 2013). The numbers of T and B cells per perivascular cuff and infiltrating the surrounding parenchyma were assessed using an Axiolmager A.1 light microscope connected to an AxioCam MRc (Carl Zeiss). The images were processed using AxioVision 4.8 software (Carl Zeiss) for detecting viral antigens and $\mathrm{T}$ and $\mathrm{B}$ lymphocytes. Fifteen cuffs (five mild, five moderate and five severe) were analysed for each brain region in each animal.

Isolation of DNA and RNA and synthesis of complementary DNA (cDNA)

Firstly, total DNA was extracted from each brain region in parallel $(200 \mu \mathrm{m})$ using the DNAzol reagent according to the manufacturer's instructions (Invitrogen, Life Technologies). Total RNA was extracted from $200-\mu \mathrm{m}$ sections of frozen tissue to quantify cytokine transcripts, using the TRIzol reagent protocol (Invitrogen). Genomic DNA contaminating the isolated RNAs was removed using Turbo DNA-free kit according to manufacturer's instructions (Ambion). In addition, isolated RNA was also precipitated by removing proteins using $3 \mathrm{M}$ sodium acetate, pH 5.5 (Ambion), glycogen $(5 \mathrm{mg} / \mathrm{mL}$ ) and ethanol. cDNA was obtained by reverse transcription of $100 \mathrm{ng}$ of purified RNA according to the Superscript III protocol (Invitrogen).

\section{Real-time PCR ( $q P C R)$}

The single-tube TaqMan assay (Life Technologies) was used; the customized primers and probes for targeting cytokine genes are detailed in Table 1 . The primers and the probe used for viral DNA detection were the same as described previously for BHV5 gC (Diallo et al., 2011; Cardoso et al., 2013).

The quantitative PCR mixtures $(50 \mu \mathrm{L})$ contained $1.2 \mu \mathrm{g}$ of viral DNA and cytokine cDNA, and $400 \mathrm{nM}$ primers and $200 \mathrm{nM}$ probes FAM-mGB (5' region) were used in qPCR initiated by sequential amplification with 40 cycles at $95^{\circ} \mathrm{C}(15 \mathrm{~s})$ and $60{ }^{\circ} \mathrm{C}$ $(60 \mathrm{~s})$. The results are expressed as viral DNA detection and as relative cytokine gene expression, indicating the fold change (up- or downregulation) in expression of the cytokine genes. Quantification of cytokine gene expression was performed using the $2^{-\Delta \Delta C t}$ method, using the bovine histone 2a gene (H2A; Bt03252057_g1) as a reference gene as described previously (Cardoso et al., 2013). The reaction was carried out and analysed using the StepOnePlus real-time instrument software (Applied Biosystems). The data were obtained from three replicates of each sample (Livak and Schmittgen, 2001).

\section{Statistical analysis}

The perivascular cuff distribution within the brain was assessed by comparing the rostral and caudal regions, and total perivascular cuffs per animal were compared among brain regions using the non-parametric exact Wilcoxon sum rank test. For $\mathrm{T}$ and $\mathrm{B}$ lymphocytes, significant differences among groups were determined using one-way ANOVA followed by Tukey's multiple comparison test. A value of $P<0.05$ was considered statistically significant. Data are expressed as the mean \pm standard deviation (SD). All statistical analyses were performed using Prism software (GraphPad v. 6.1).

\section{Results}

\section{Histopathology and immunohistochemistry findings}

Histopathological findings in the brains of 38 cases of acute BHV5 neurological infection were mainly composed of foci of perivascular cuffing with mononuclear cells, mainly lymphocytes (Figs. 1A-D). Perivascular cuffings were observed in the Obs (Fig. 1A), the frontal, temporal and parietal lobes (Figs. 1B, C), and in the piriform lobe (Fig. 1D). These changes ranged in composition, varying from a few cells (Figs. 1C, D) to multiple layers of cell nuclei in the VirchowRobin spaces (Figs. 1A-C).

Lymphocyte infiltration into the brain parenchyma was rarely observed. Perivascular cuffing was severe in the Obs, piriform lobe, hippocampus, and frontal, temporal and parietal lobes (Fig. 2A). There appeared to be more cuffs in the rostral regions than the caudal

Table 1

Names, descriptions, and locations of the (Bos taurus) genes evaluated by qRT-PCR.

\begin{tabular}{|c|c|c|c|}
\hline Gene symbol & ID & Description & Location \\
\hline INFAC & 281.236 & Interferon alpha C (INF-alpha C) & Chromosome 8, AC_000.165.1 \\
\hline INFB1 & 281.845 & Interferon, beta 1 , fibroblast & Chromosome 8, AC_000.165.1 \\
\hline INFG & 281.237 & Interferon, gamma & Chromosome 5, AC_000.162.1 \\
\hline IL2 & 280.822 & Interleukin 2 & Chromosome 17, AC_000.174.1 \\
\hline IL4 & 280.824 & Interleukin 4 & Chromosome 7, AC_000.209.1 \\
\hline IL6 & 517.016 & Interleukin 6 & Chromosome 8, AC_000.162.1 \\
\hline IL1F10 & 615.702 & Interleukin 1 , family member 10 & Chromosome 11, AC_000.168.1 \\
\hline TNF & 280.943 & Tumour necrosis factor (TNF alpha) & Chromosome 23, AC_000.180.1 \\
\hline$L T B R$ & 280.845 & Lymphotoxin beta receptor (TNF superfamily member 3) & Chromosome 5, AC_000.162.1 \\
\hline
\end{tabular}




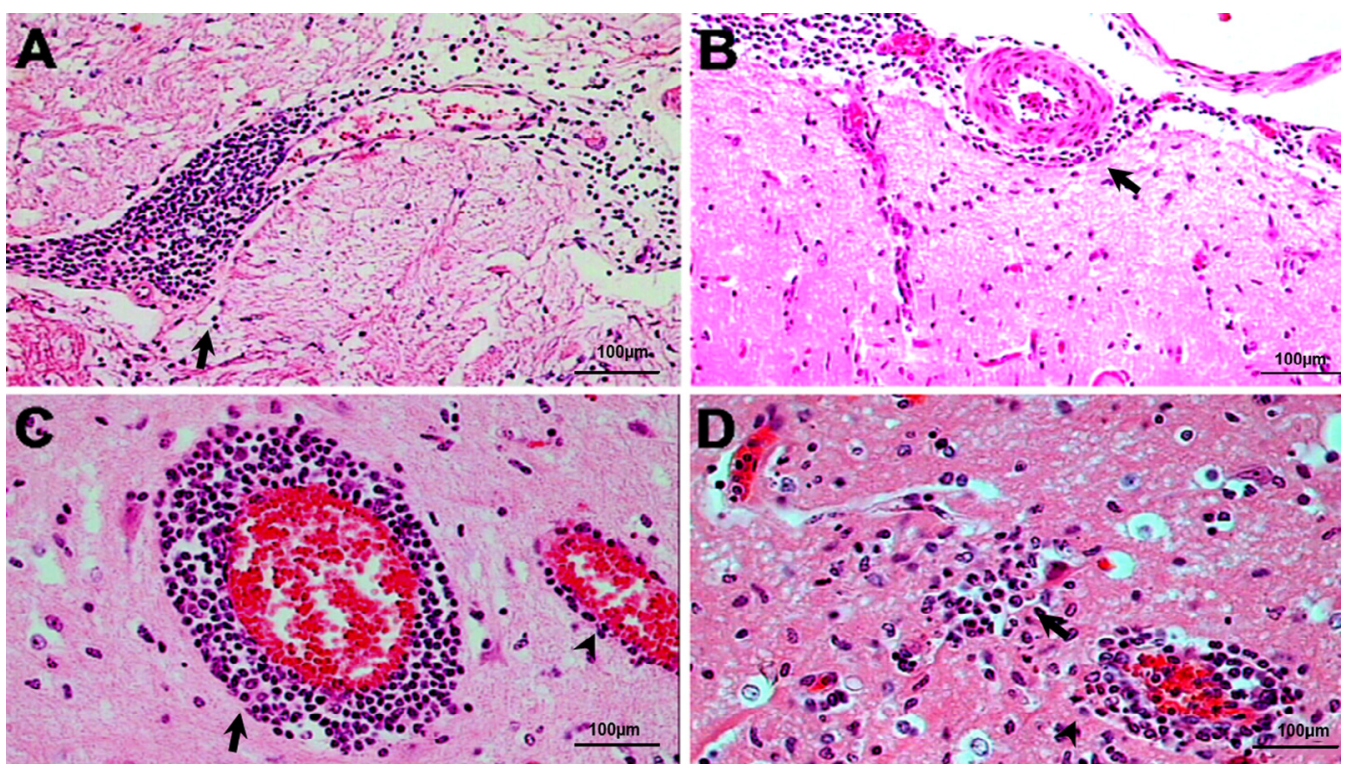

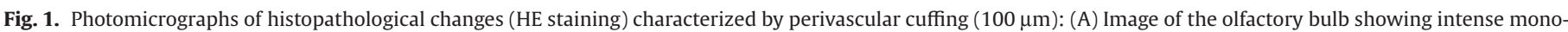

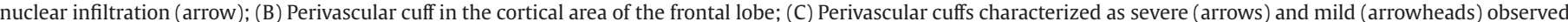
in the frontal, temporal and parietal lobes; (D) Perivascular cuffs considered moderate (arrows) localized in the hippocampus.

regions $(P<0.005)$. Viral antigens were detected in the same regions (Fig. 2B). A significant correlation was observed between severe perivascular cuffs and BHV5 antigen distribution $(r=0.947 ; P<0.005)$. Viral antigens and genomic DNA were mainly detected in the Obs and piriform lobe, simultaneously $(r=0.987 ; P<0.005)$.

Inflammatory infiltrates of CD3+ lymphocytes were detected mainly in the Obs, hippocampus, and piriform, frontal, temporal and parietal lobes (Figs. 3A, B). A strong association between CD3+ lymphocytes and BHV5 antigens was observed in the $\mathrm{Ob}$ and the frontal, temporal, and parietal lobes (Figs. 4A-D). CD45R+ cells (approximately 10\%) were observed only in the temporal and parietal lobes (Figs. 4E, F).

Pro-inflammatory and anti-inflammatory profiles associated with virus distribution

The symbols, identities, descriptions and locations of genes studied are presented in Table 1. In this study, the highest expression levels of the genes INFG and BHV5 gC were observed in the Obs and hippocampus and in the piriform, frontal temporal and parietal lobes (Fig. 5A; $P<0.005)$. INFAC and INFB1 expression was similar to control samples (Fig. 5). The genes IL4, IL2 and BHV5 gC were detected and positively correlated in the Obs, hippocampus and piriform lobe (Fig. 5B; $P<0.005$ ). The parietal cortex had higher expression of IL4 than the other cytokines or BHV5 gC, which were detected at low levels (Fig. 5B; $P<0.005$ ). In the Obs, piriform lobe, hippocampus and parietal lobe, the expression levels of TNF, LTBR and BHV5 gC were approximately equal, with lower levels observed in the frontal and temporal cortices (Fig. 5C; $P<0.005$ ).

\section{Discussion}

This study demonstrated the presence of severe inflammatory infiltrates and the expression of the pro-inflammatory cytokine genes IFNG, IL2, TNF and LTBR in the brains of cattle acutely infected with BHV5. These findings were positively correlated with the presence of BHV5 DNA genome and viral antigens in the forebrain region.
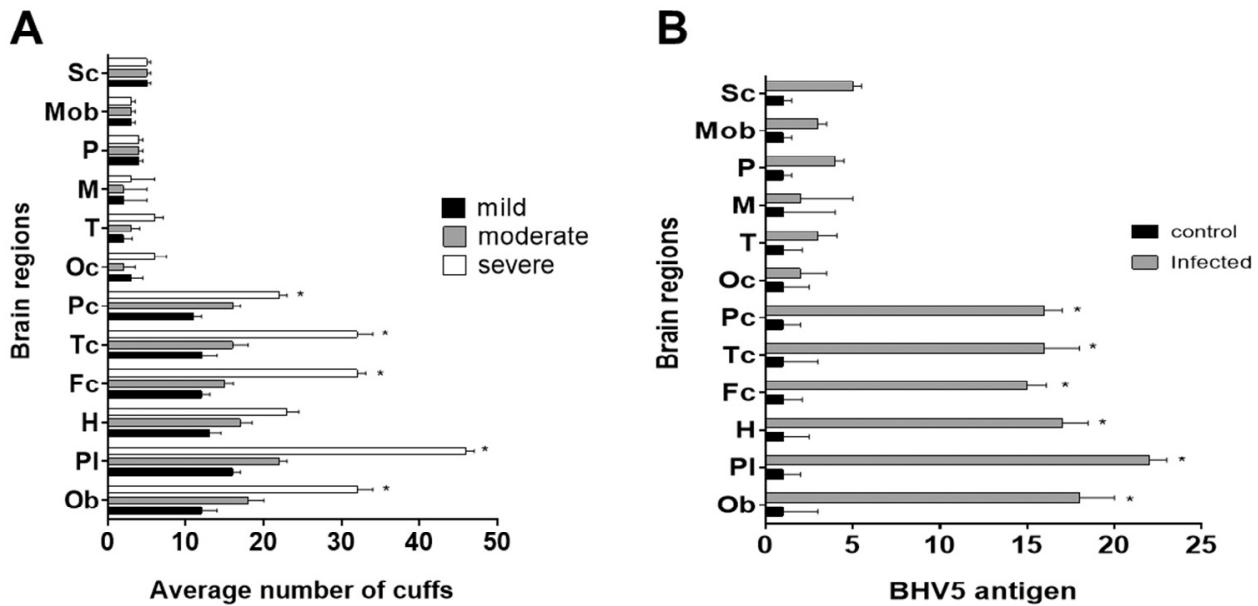

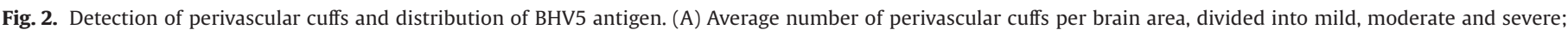
(B) Frequency of detection of BHV5 antigen by immunohistochemistry per brain area. The mean value was statistically significant ( ${ }^{*} P<0.005$ ) 


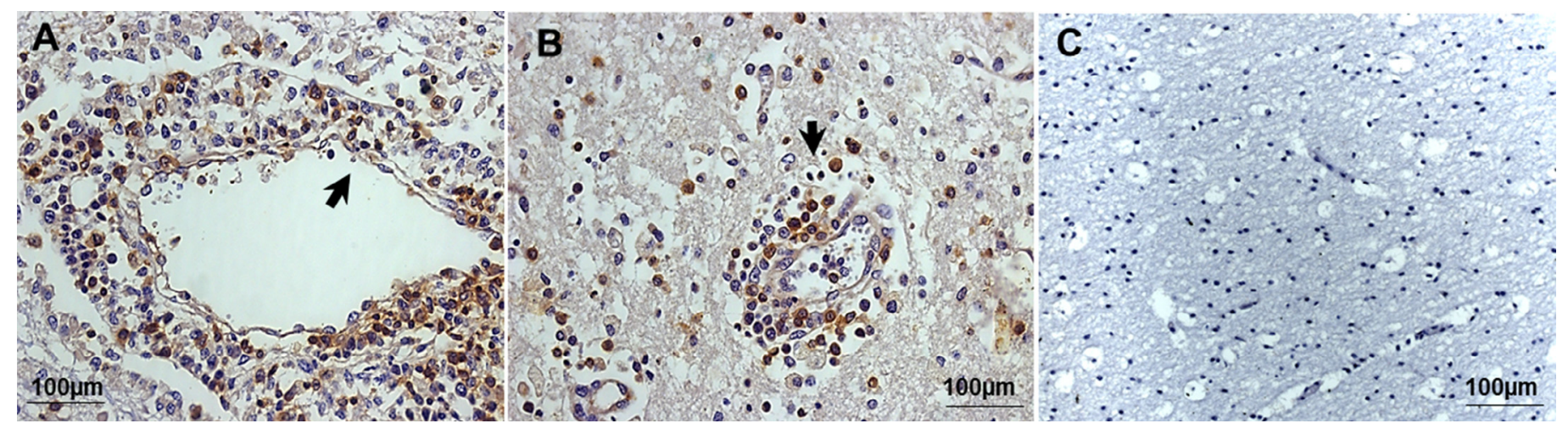

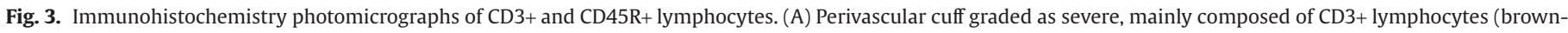

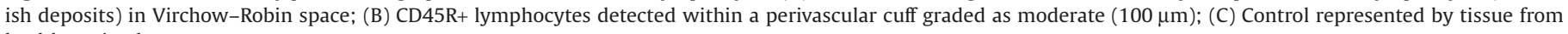
healthy animals.

The histopathological findings were similar to previous reports on the pathogenesis of BHV5 in experimentally infected rabbits (Chowdhury et al., 1997; Lee et al., 1999; Dezengrini et al., 2009; Machado et al., 2013). However, in contrast to the present study, which examined only brains of acutely diseased animals, some of the BHV5-infected rabbits did not develop neurological signs (Machado et al., 2013). In the rabbit model, microscopic examination revealed histological changes in the cerebral cortices (frontal, temporal, occipital, parietal and piriform lobes) characterized by mononuclear perivascular cuffs (Dezengrini et al., 2009; Machado et al., 2013). In another study, it was postulated that asymptomatic encephalitis in calves experimentally infected with BHV5 might be associated with mild foci of inflammatory cells (Isernhagen et al., 2011). However, in rabbits presenting with neurological signs, BHV5 antigens were also detected in the cerebellum, a finding not observed in the present study. Regarding CD45R+ lymphocytes, 10\%
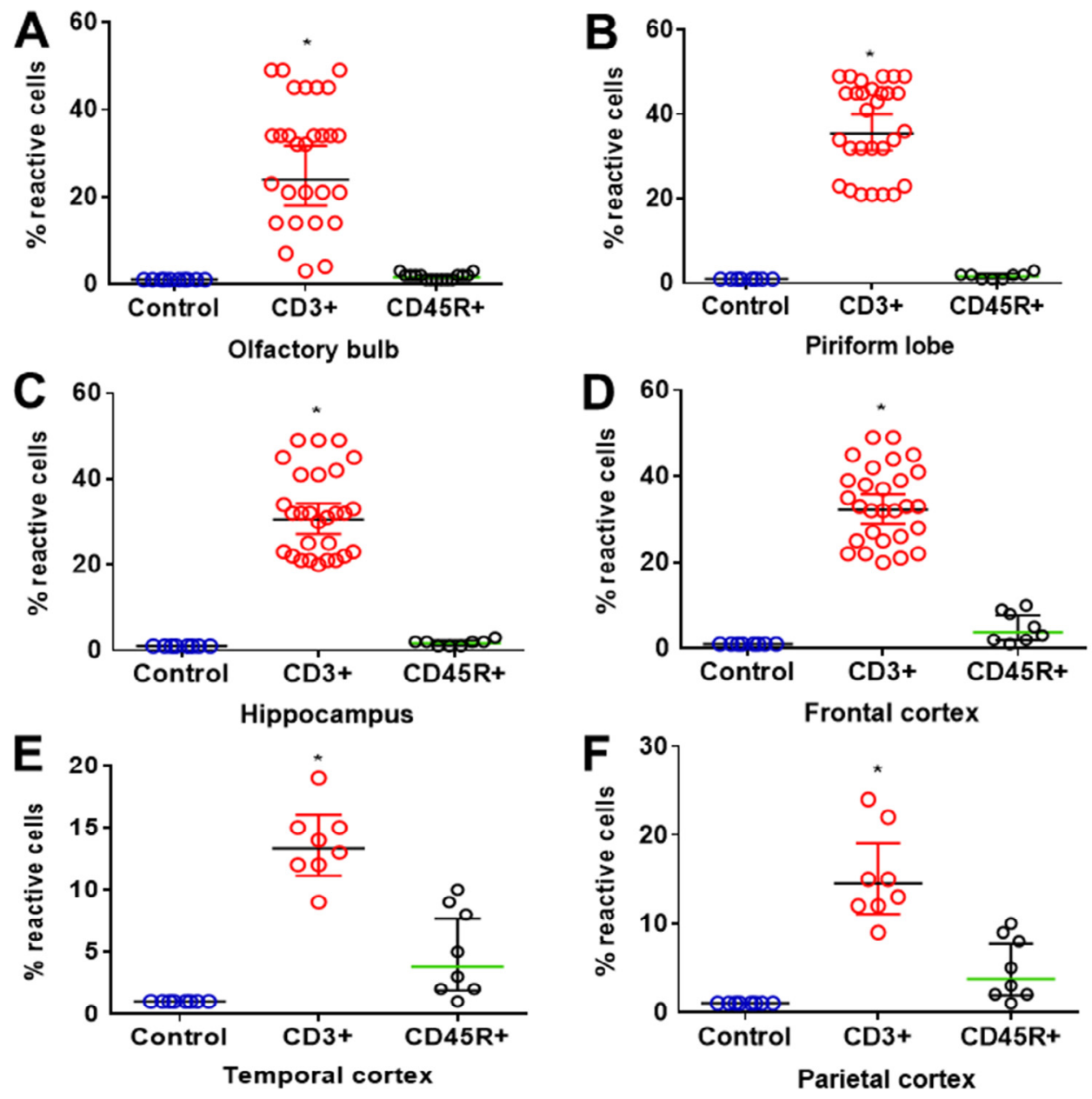

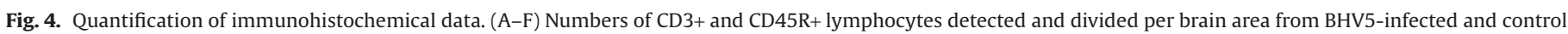
animals. The mean value was statistically significant $\left({ }^{*} P<0.005\right)$. 

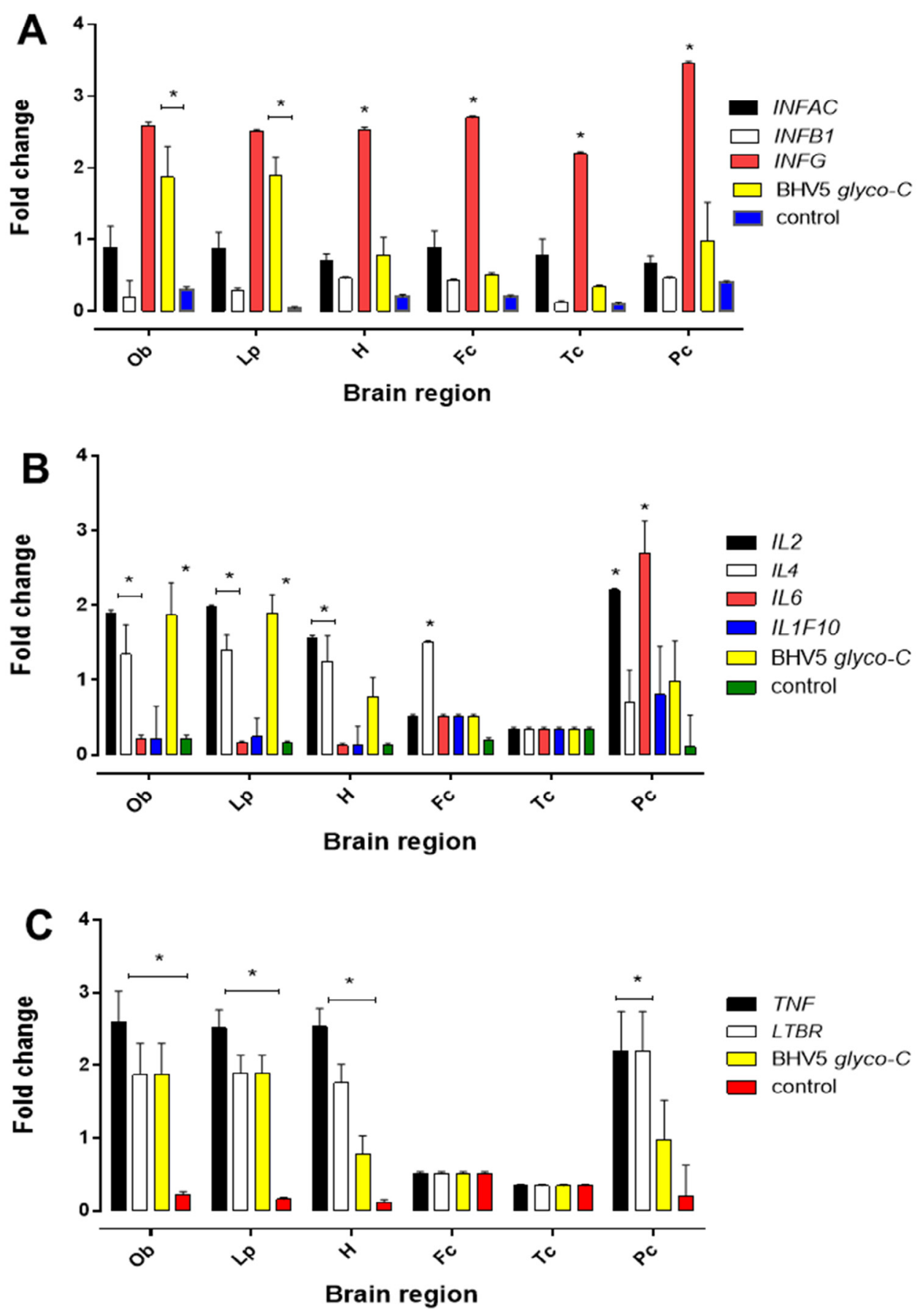

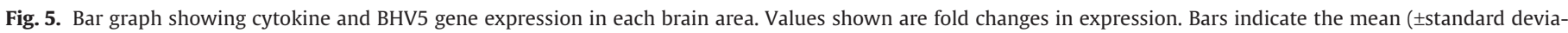

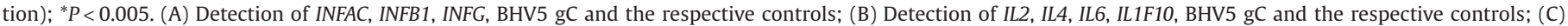
Detection of TNF, LTBR, BHV5 gC and the respective controls.

of positive cells were observed only in the temporal and parietal lobes, a finding similar to that of a previous study (Machado et al., 2013). Similarly, acute replication of HSV1 in the trigeminal ganglia is largely controlled by the innate immune system, including macrophages and activated lymphocytes (Griffin et al., 2010; St Leger and Hendricks, 2011; Piret and Boivin, 2015).

All animals examined in this study died during the acute phase of BHV5-associated neurological disease. Molecular analysis revealed that the Obs and piriform lobe had the highest level of BHV5 gC gene expression $(P<0.005)$. Previous studies have demonstrated uniform distribution of the BHV5 genomes among brain areas in experimentally infected rabbits (Mayer et al., 2006; Machado et al., 2013). In another study, rabbits acutely infected with BHV5 harboured viral DNA mostly in the trigeminal ganglia, cerebellum, anterior cortex and pons-medulla (Dezengrini et al., 2009). However, in experimentally infected calves, BHV5 DNA was detected in the trigeminal ganglia, midbrain, thalamus and Obs (Vogel et al., 2003). Additionally, similar BHV1 viral genome distribution in the CNS of infected calves has been reported (Silva et al., 2007; Marin et al., 2015). In all animals studied, whether naturally or experimentally infected, BHV5 has most consistently been identified in the Obs. In general, latency and reactivation are critical strategies for the 
survival of herpesviruses in nature (Jones, 2003). Typically, most cells infected by both human and animal alphaherpesviruses support lytic replication, while only specific cell subsets, especially neurons, harbour latent virus (Jones, 2003; Griffin et al., 2010).

A recent study described weak $T$ cell infiltration associated with inefficient virus clearance in HSV1 and West Nile virus neuroinflammation (Franco and Fernández-Suárez, 2015). Moreover, cuffs composed of large numbers of $\mathrm{T}$ cells could result in cytotoxicity and, as a consequence, in CNS damage (Xie and Yang, 2015). Several reports have demonstrated that viral encephalitis leads to vigorous cytokine expression, an increase in BBB permeability related to IFN- $\gamma$ release and stimulation of T cell activity, thereby increasing viral clearance (Sarma, 2014). Cytokines play an essential role in modulation of the host immune response, especially in the pathogenesis of many viral infections (Franco and Fernández-Suárez, 2015). Herein, the neuroinflammatory process induced by BHV5 infection was marked by up-regulation of the cytokines INFG, IL2, TNF and LTBR.

The importance of interferons (IFNs) in the anti-viral immune response is well known (St Leger and Hendricks, 2011). Instead of a direct antiviral effect, IFNs induce the expression of effector proteins, which, in turn, interfere with or inhibit various stages of viral replication, assembly or release (Piret and Boivin, 2015). The balance between inflammatory and anti-inflammatory cytokines is crucial for avoiding damage to neural tissue (Piret and Boivin, 2015). It is reasonable that the expression of these cytokines would somehow suppress the immune response and contribute to viral spread. Several studies have concluded that a cell-mediated immune response persists in the trigeminal ganglia of mice latently infected with HSV1 or in rodents infected with HSV2 (Jones, 2003; St Leger and Hendricks, 2011). Persistence of immune cells in the trigeminal ganglia is important for maintaining latency, because CD8+ T cells that produce INF- $\gamma$ can prevent reactivation from latency (Perez et al., 2006; St Leger and Hendricks, 2011).

The expression of IL2, a T cell growth factor mainly synthesized by CD4+ lymphocytes and to a lesser extent by CD8+ cells, which also acts as an autocrine growth factor, was observed in this study. Chemokines cause the accumulation and activation of leukocytes in tissues (Yshii et al., 2015), which are essential for inflammation and the host response to infection (Crouse et al., 2015). Recently, the importance of CNS-resident cells in the establishment of an effective inflammatory response to BHV5 infection has been reported (Marin et al., 2014). Moreover, the present study also demonstrated an upregulation of TNF (TNF- $\alpha$ ) and $L T B R$ (TNF- $\beta$ ) gene expression. Both cytokines present similar activity; however, TNF- $\beta$ stimulates phagocytosis, increasing the synthesis of nitrogen oxide (NO; Xie and Yang, 2015). Although NO was not measured in this study, a previous report demonstrated its production in the CNS of BHV5-infected rabbits (Dezengrini et al., 2009).

\section{Conclusions}

These findings provide additional evidence that BHV5 neurological infection in cattle is associated with severe inflammatory infiltration, predominantly by CD3+ cells, followed by proinflammatory cytokine expression, that correlates spatially with the distribution of viral DNA and antigens. Regardless, further studies are needed to demonstrate the cytokine profile and involvement in the fatal outcome of BHV5-induced neurological infection in cattle.

\section{Conflict of interest statement}

None of the authors of this paper has a financial or personal relationship with other people or organizations that could inappropriately influence or bias the content of the paper.

\section{Acknowledgements}

The authors thank the Fundação de Amparo à Pesquisa do Estado de São Paulo (Grant 2012/16715-6) for their support. EF Flores and TC Cardoso are recipients of CNPq (Brazilian Council for Research) fellowships and funding. The BHV5 monoclonal antibody (BHV5 2F9) was kindly provided by Prof. Eduardo Furtado Flores.

\section{References}

Cardoso, T.C., Ferrari, H.F., Garcia, A.F., Bregano, L.C., Andrade, A.L., Nogueira, A.H., 2010. Immunohistochemical approach to the pathogenesis of clinical cases of bovine herpesvirus type 5 infections. Diagnostic Pathology 5, 57.

Cardoso, T.C., Silva-Frade, C., Taparo, C.V., Okamura, L.H., Flores, E.F., 2013. Validation of a reference control for an SYBR-Green fluorescence assay-based real-time PCR for detection of bovine herpesvirus 5 in experimentally exposed bovine embryos. Molecular and Cellular Probes 27, 237-242.

Chowdhury, S.I., Lee, B.J., Moiser, D., Sur, J.H., Osorio, F.A., Kennedy, G., Weiss, M.L. 1997. Neuropathology of bovine herpesvirus type 5 (BHV-5) meningoencephalitis in a rabbit seizure model. Journal of Comparative Pathology 117, 295-310.

Crouse, J., Kalinke, U., Oxenius, A., 2015. Regulation of antiviral T cell responses by type I interferons. Nature Reviews. Immunology 15, 231-242.

Davison, A.J., 2010. Herpesvirus systematics. Veterinary Microbiology 143, 52 69.

Davison, A.J., Eberle, R., Ehlers, B., Hayward, G.S., McGeoch, D.J., Minson, A.C., Pellett, P.E., Roizman, B., Studdert, M.J., Thiry, E., 2009. The order Herpesvirales. Archives of Virology 154, 171-177.

Del Medico Zajac, M.P., Ladelfa, M.F., Kotsias, F., Muylkens, B., Thiry, J., Thiry, E., Romera, S.A., 2010. Biology of bovine herpesvirus 5. The Veterinary Journal 184, $138-145$.

Dezengrini, R., Weiss, M., Torres, F.D., Oliveira, M.S., Furian, F., Mello, C.F., Weiblen, R., Flores, E.F., 2009. Bovine herpesvirus 5 induces an overproduction of nitric oxide in the brain of rabbits that correlates with virus dissemination and precedes the development of neurological signs. Journal of Neurovirology 15, 153-163.

Diallo, I.S., Corney, B.G., Rodwell, B.J., 2011. Detection and differentiation of bovine herpesvirus 1 and 5 using a multiplex real-time polymerase chain reaction. Journal of Virological Methods 175, 46-52.

Favier, P.A., Marin, M.S., Moran, P.E., Odeon, A.C., Verna, A.E., Perez, S.E., 2014. Latency of bovine herpesvirus type 5 (BoHV-5) in tonsils and peripheral blood leukocytes. The Veterinary Journal 202, 134-140.

Ferrari, H.F., Luvizotto, M.C.R., Rahal, P., Cardoso, T.C., 2007. Detection of bovine Herpesvirus type 5 in formalin-fixed, paraffin-embedded bovine brain by PCR: A useful adjunct to conventional tissue-based diagnostic test of bovine encephalitis. Journal of Virological Methods 146, 335-340.

Franco, R., Fernández-Suárez, D., 2015. Alternatively activated microglia and macrophages in the central nervous system. Progress in Neurobiology 131, 65-86.

Griffin, B.D., Verweij, M.C., Wiertz, E.J.H.J., 2010. Herpesviruses and immunity: The art of evasion. Veterinary Microbiology 143, 89-100.

Isernhagen, A.J., Cosenza, M., da Costa, M.C., Medici, K.C., Balarin, M.R.S., Bracarense, A.P.F.R.L., Alfieri, A.A., Lisboa, J.A.N., 2011. Asymptomatic encephalitis in calves experimentally infected with bovine herpesvirus-5. Canadian Veterinary Journal $52,1312-1318$

Jones, C., 2003. Herpes simplex virus type 1 and bovine herpesvirus 1 latency. Clinical Microbiology Reviews 16, 79-95.

Jones, C., da Silva, L.F., Sinani, D., 2011. Regulation of the latency-reactivation cycle by products encoded by the bovine herpesvirus 1 (BHV-1) latency-related gene. Journal of Neurovirology 17, 535-545.

Lee, B.J., Weiss, M.L., Moiser, D., Chowdhury, S.I., 1999. Spread of bovine herpesvirus type 5 (BHV-5) in rabbit brain after intranasal inoculation. Journal of Neurovirology 5, 474-484.

Livak, K., Schmittgen, T., 2001. Analysis of relative gene expression data using real-time quantitative PCR and the 2(-Delta Delta C(T) method. Methods: A Companion to Methods in Enzymology 25, 402-408.

Lovato, L., Inman, M., Henderson, G., Doster, A., Jones, C., 2003. Infection of cattle with a bovine herpesvirus 1 strain that contains a mutation in the latency-related gene leads to increased apoptosis in trigeminal ganglia during the transition from acute infection to latency. Journal of Virology 77, 4848-4857.

Machado, G.F., Bernardi, F., Hosomi, F.Y.M., Peiro, J.R., Weiblen, R., Roehe, P.M., Alessi, A.C., Melo, G.D., Ramos, A.T., Maiorka, P.C., 2013. Bovine herpesvirus-5 infection in a rabbit experimental model: Immunohistochemical study of the cellular response in the CNS. Microbial Pathogenesis 57, 10-16.

Marin, M.S., Quintana, S., Leunda, M.R., Odeon, A.C., Perez, S.E., 2014. Toll-like receptor expression in the nervous system of bovine alpha-herpesvirus-infected calves. Research in Veterinary Science 97, 422-429.

Marin, M.S., Leunda, M.R., Verna, A.E., Moran, P.E., Odeon, A.C., Perez, S.E., 2016 Distribution of bovine herpesvirus type 1 in the nervous system of experimentally infected calves. The Veterinary Journal 209, 82-86.

Mayer, S.V., Quadros, V.L., Vogel, F.S., Winkelmann, E.R., Arenhart, S., Weiblen, R., Flores, E.F., 2006. Dexamethasone-induced reactivation of bovine herpesvirus type 5 latent infection in experimentally infected rabbits results in a broader distribution of latent viral DNA in the brain. Revista brasileira de pesquisas médicas e biológicas 39, 335-343. 
Perez, S., Lovato, L., Zhou, J., Doster, A., Jones, C., 2006. Comparison of inflammatory infiltrates in trigeminal ganglia of cattle infected with wild-type bovine herpesvirus 1 versus a virus strain containing a mutation in the LR (latencyrelated) gene. Journal of Neurovirology 12, 392-397.

Perez, S.E., Bretschneider, G., Leunda, M.R., Osorio, E.A., Flores, E.F., Odeon, A.C., 2002. Primary infection, latency, and reactivation of bovine herpesvirus type 5 in the bovine nervous system. Veterinary Pathology 39, 437-444.

Piret, J., Boivin, G., 2015. Innate immune response during herpes simplex virus encephalitis and development of immunomodulatory strategies. Reviews in Medical Virology 25, 300-319.

Sarma, J.D., 2014. Microglia-mediated neuroinflammation in an amplifier of virusinduced neuropathology. Journal of Neurovirology 20, 122-136.

Silva, M.S., Brum, M.C., Loreto, E.L., Weiblen, R., Flores, E.F., 2007. Molecular and antigenic characterization of Brazilian bovine herpesvirus type 1 isolates recovered from the brain of cattle with neurological disease. Virus Research 129 191-199.

St Leger, A.J., Hendricks, R.L., 2011. CD8+ T cells patrol HSV-1-infected trigeminal ganglia and prevent viral reactivation. Journal of Neurovirology $17,528-$ 534.

Vogel, F.S., Caron, L., Flores, E.F., Weiblen, R., Winkelmann, E.R., Mayer, S.V., Bastos, R.G., 2003. Distribution of bovine herpesvirus type 5 DNA in the central nervous systems of latently, experimentally infected calves. Journal Clinical Microbiology 41, 4512-4520.

Xie, L., Yang, S.-H., 2015. Interactions of astrocytes and T cells in physiological and pathological conditions. Brain Research 1623, 63-73.

Yshii, L., Gebauer, C., Bernard-Valnet, R., Liblau, R., 2015. Neurons and T cells: Understanding this interaction for inflammatory neurological diseases. European Journal of Immunology 45, 2712-2720. 